\title{
A web-based early-warning service to monitor drinking-water treatment plant operations
}

\author{
Franclin S. Foping
}

Cork Constraint Computation Centre, UCC

\section{Introduction}

Drinking contaminated water can be harmful to our health. According to the World Health Organization, about 1.8 million people die every year across the world from water-borne diseases mainly caused by polluted drinking water. Furthermore, the cryptosporidium outbreak that happened in Galway in 2007 indicates the urgency to provide appropriate solutions in order to counteract this ominous situation in the country.

Water treatment plants (WTP) are basic components of modern water supply and distribution systems. These are engineering systems that purify raw water to specific safety levels. The raw water passes through a series of treatment phases wherein it is processed and purified according to existing safety protocols regulating drinking water. After undergoing a purification step, the drinking water is distributed to the consumers through a network of pipes, pumps and reservoirs. The research presented in this report is focused on the safety of these critical infrastructures. In particular, the goal is to develop a novel Web-based early warning system (EWS) for monitoring and assessing the safety level of WTP.

EWS have been used for many years in the drinking water industry with the objective of timely detect the presence of harmful chemical, microbiological, biological and radiological agents in the water. However, the presence of harmful agents in the water, on its own, does not provide a complete explanation of drinking water disasters. This is because malicious agents are just one parameter used to assess the safety of a system.

The idea of a new Web-based EWS will be presented briefly in this article. Based on it, WTP are viewed as components of a complex socio-technical system. The EWS, which is currently under development, will be able to receive inputs from its users, who are working in different agencies and WTP, as well as from sensors. Then, it will assess the safety level of WTP and finally it will dispatch warning messages whenever the safety health of WTP is above a predefined threshold. 


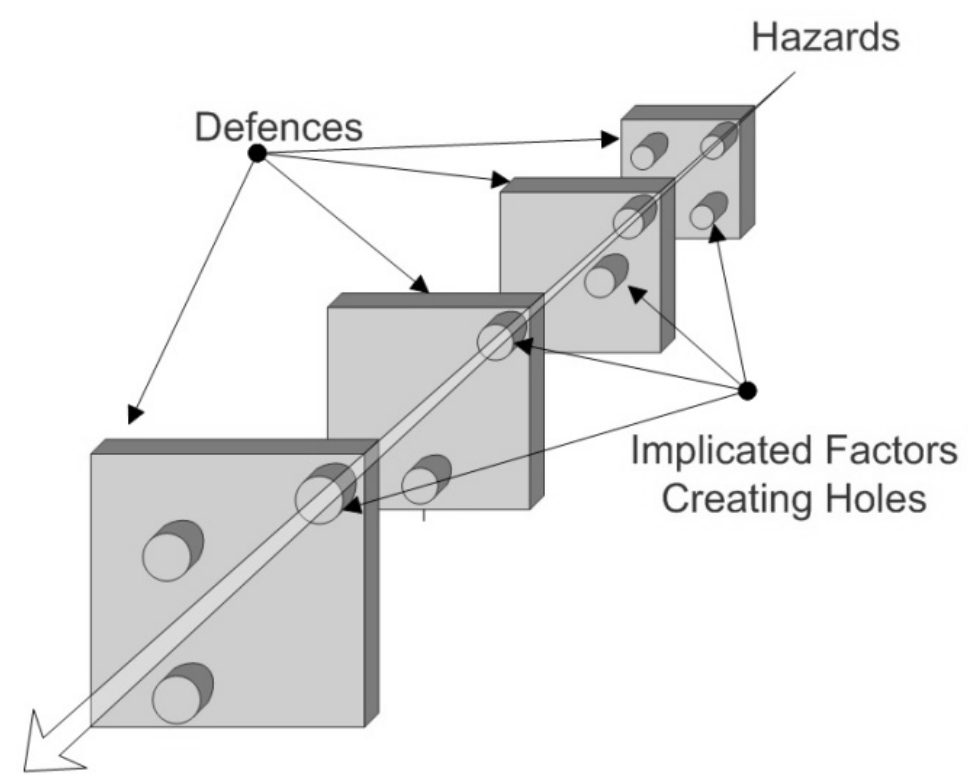

Accident or Loss

Figure 1: The "Swiss Cheese" Model

\section{Problem Statement}

The work presented here is based on the assumption that failures and accidents in WTP can be better explained by using the tenets of the socio-technical theory. This means that WTP and their associated EWS are not just technical entities. In contrast, they are components embedded in larger social and organizational networks, which all together form a complex socio-technical system. In order to understand the origin of failures in a typical complex system, an analogy was presented by James Reason in the early 1990s: the "Swiss-Cheese" model, which is depicted in Figure 1. According to this model, hazards are prevented from bringing about accidents by a sequence of safeguards, each of which may have some holes in them, thereby making the entire system more vulnerable. It should be noted that the presence of a hole in a barrier at a given time depends on implicated factors such as active failures and latent conditions (i.e, actions and/or decisions that may have unforeseen consequences in the future). In addition, the holes are in constant flux. Thus, an accident can happen if by any chance the holes are aligned so that a hazard can traverse the entire system from one point to another.

The "Swiss Cheese" Model Given the diversity of the users in terms of their geographic location and their role in the socio-technical system, two key questions need to be answered:

1. What access mechanism of our system will be available to end-users?

2. Another concern raised by the provider is related to mechanisms and policies that will have to be devised in order to handle the diversity of users.

The most popular method for deploying an application is to record it on a CD/DVD and 
dispatch it to customers. They will need to install it on their computers and run it from there. Because of this, the service provider (application owner) will have to send another copy of the application every time a patch is released or a new version of the application has been released. This process could be very cumbersome if the number of customers is significantly high. Also, customers will need to possess up-to-date computers equipped with advanced and cutting-edge hardware, as some functionalities of the applications may be disabled if their computers do not meet the minimal requirements in terms of processing power, e.g, if the computer is short of memory or disk storage. This kind of application is known as on-premises software.

Another interesting alternative to deploy our system may consist of making it accessible from a Web browser such as Mozilla Firefox, Google Chrome or Internet Explorer. Such applications include Google's GMail which allows one to check, print and reply to emails online without having to install any additional software on one's computers. In order to run these types of applications, only a Web browser and an active Internet connection are required and there is no requirement for a dedicated or above average computer. These types of applications address several issues that were raised by their on-premises counterparts. For example, updating a Web application is a swift and straightforward process as it is only performed on the provider's computers, not on customers' premises. Another advantage is that there is no need to dispatch a CD or a DVD of the application to potential customers, all they have to do is log on to the provider's Website and use the software online. This kind of application is referred to as software as a service. This category of application also provides a valuable answer to the second question raised above. Indeed, software as a service applications have a better return on investment, making them very appealing. In addition, software as a service applications can handle several users simultaneously. They can then be seen as a "one size fits all" solution. From a provider's viewpoint, it means that a software as a service application can serve several users regardless their geographical locations. Table 1 summarizes the different characteristics of two types of applications discussed in this section.

In this project, we propose to build a novel type of EWS that will be accessible from the Internet using the software as a service deployment model, due to the complexity of our socio-technical system, which involves several users located throughout the country. Figure 2 shows an overview of our socio-technical system. A given WTP is just a small component, albeit a key one of the overall system. Several interactions can be noticed in the socio-technical system. For instance, a WTP is regulated by the water service authority that is part of a local authority. The local authority is ultimately responsible for the WTP, which is supervised and audited by an inspector of the Environmental Protection Agency (EPA). These simple use cases illustrate the extensive amount of collaboration being carried out in our system, as well as its level of complexity. In short, the software as a service deployment model was selected for our EWS because we want to bring together all parties involved in the treatment process for drinking water in this country. 
Table 1: Characteristics of the two types of application

\begin{tabular}{l|ll}
\hline Cost & Software as a service & On-premises software \\
\hline License & $\begin{array}{l}\text { Relatively cheaper, } \\
\text { sometimes free when the } \\
\text { provider relies on } \\
\text { advertisement }\end{array}$ & Usually more expensive \\
\hline Accessibility & $\begin{array}{l}\text { You only pay for what you } \\
\text { have used }\end{array}$ & $\begin{array}{l}\text { An upfront license fee is } \\
\text { required }\end{array}$ \\
\hline $\begin{array}{l}\text { Software } \\
\text { requirements }\end{array}$ & $\begin{array}{l}\text { Normally there is no need to } \\
\text { install any software to run it }\end{array}$ & $\begin{array}{l}\text { Yes, that is why we } \\
\text { purchased an upfront license } \\
\text { fee }\end{array}$ \\
\hline $\begin{array}{l}\text { Hardware } \\
\text { requirements }\end{array}$ & $\begin{array}{l}\text { Minimal. The bulk of the } \\
\text { application can be run online }\end{array}$ & $\begin{array}{l}\text { Yes, because the application } \\
\text { runs from your computer }\end{array}$ \\
\hline Update process & $\begin{array}{l}\text { Very easy as it is performed } \\
\text { on the provider's premises }\end{array}$ & $\begin{array}{l}\text { Cumbersome as the } \\
\text { customer is ultimately } \\
\text { responsible for that }\end{array}$ \\
\hline $\begin{array}{l}\text { Multi-users } \\
\text { simultaneously }\end{array}$ & $\begin{array}{l}\text { Yes, thanks to its } \\
\text { "one-size-fits-all" feature. }\end{array}$ & $\begin{array}{l}\text { Normally only one user can } \\
\text { run the application at a time. }\end{array}$ \\
\hline
\end{tabular}

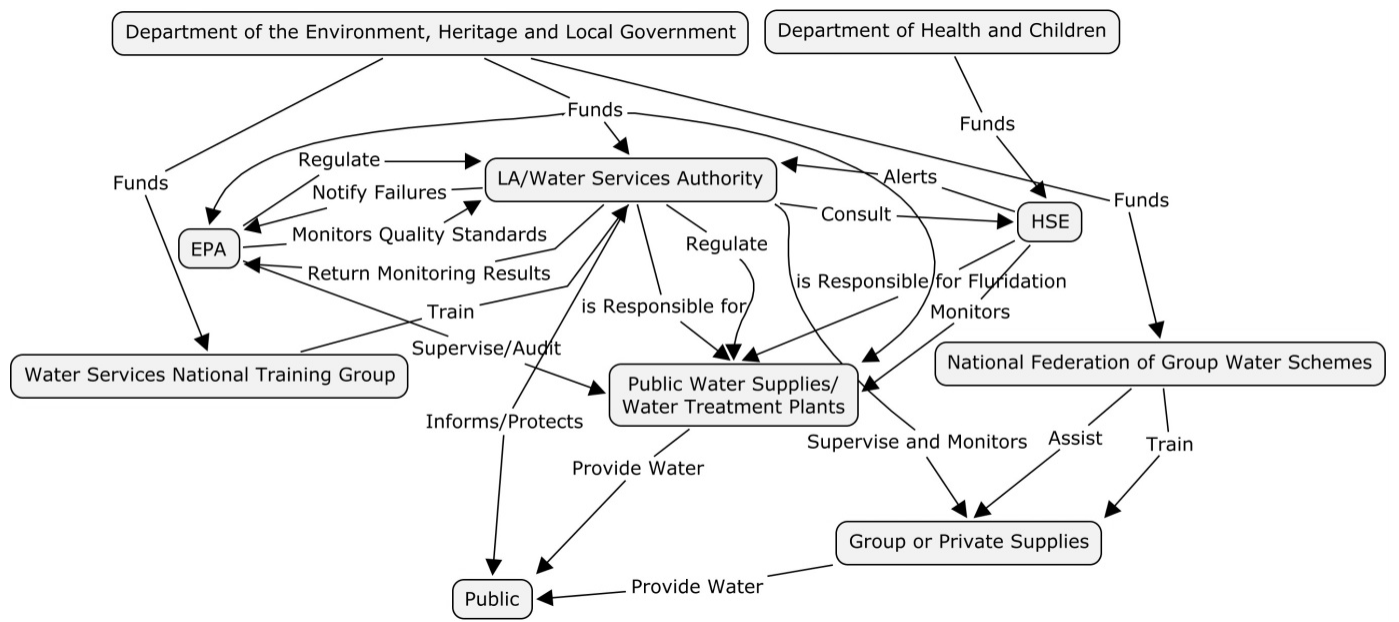

Figure 2: An overview of the entire system 


\section{Project Goals}

The ultimate objective of this Ph.D. research is to make use of the latest Web technologies to build a Web platform for a new type of EWS. The aim of this novel EWS is to be used by organizations that altogether form the governance model of the drinking water treatment and distribution system in the Republic of Ireland, such as Local Authorities, WTP personnel, the Health Service Executive and the Irish EPA.

The system, which is currently under development, will first receive inputs from users working in different agencies and WTP, as well as from sensors. Then will assess the safety level of WTP and finally it will dispatch warning messages whenever the safety health of WTP falls above a threshold. Our system will also show a visual representation of all WTP and their current working conditions, powered by Google Maps.

\section{Conclusion}

This paper briefly discussed some characteristics of a novel EWS for WTP that will be deployed over the Internet. The EWS will not only detect the presence of holes in the safeguard as shown in Figure 1, but also provide an explanation of the presence of these holes. To the best of our knowledge, this will be the first such EWS to be deployed over the Internet. Another research goal of our project will be to clarify the relationship between Web technologies used to deploy applications. Several other issues regarding security will be tackled in our research project. The EWS is currently in the implementation phase and we expect to complete the first version of the prototype shortly.

Thanks to my supervisor Dr. loannis Dokas and the EPA for supporting this research project. 